\title{
ANOTHER INTERESTING PROPERTY CONCERNING THE PROBABILITY MEASURES ON THE RATIONALS
}

\author{
K. W. LANE ${ }^{1}$
}

\begin{abstract}
Let $X$ be a perfect, complete, separable metric space and $P(X)$ denote the space of Borel probability measures on $X$ equipped with the topology of weak convergence. If $Y$ is a countable dense subset of $X$ then $P(Y)$ is not a $G_{\delta \text { n }}$ subset of $P(X)$. Furthermore if $X$ is separable, complete and metric, and $Y \subseteq X$. and $P(Y)$ is a $G_{\delta \sigma}$ subset of $P(X)$, then $P(Y)$ is in fact a $G_{\delta}$ subset of $P(X)$.
\end{abstract}

I. Introduction. Let $X$ be a separable metric space and $P(X)$ denote the space of Borel probability measures on $X$. The topology on $P(X)$ is the topology of weak convergence, i.e. $\lim m_{i}=m$ iff $\lim \sup m_{i}(F) \leqslant m(F)$ for all closed $F \subseteq X$. Equivalent versions of the topology and other properties of $P(X)$ can be found in [4, pp. 39-50].

In [5] Preiss showed that if $Y$ is taken to be the rationals (or more generally is first category in itself) then $Y$ is not Prohorov. Preiss then used this result to show that if $Y$ is any separable metric coanalytic space, then $Y$ is Prohorov implies that $Y$ is topologically complete (which is equivalent to $P(Y)$ being topologically complete).

In this paper it is shown that if $Y$ is a countable dense subset of a perfect Polish space $X$, then $P(Y)$ is not a $G_{\delta \sigma}$ subset of $P(X)$. Then, using Theorem 2 of [5], it is shown that if $Y \subseteq X$ (Polish) and $P(Y)$ is a $G_{\delta \sigma}$ subset of $P(X)$, then $P(Y)$ is in fact a $G_{\delta}$ subset of $P(X)$.

This result is of the same flavor as that of Luther in [3] in which he showed that local compactness of $P(Y)$ (actually the existence of a compact neighborhood) implies that $P(Y)$ is compact. It is not difficult using Luther's result to show if $P(Y)$ is $\sigma$-compact then $P(Y)$ is compact.

These three results can be summarized as follows: if $Y$ is separable and metric then:

(1) if $Y$ is $C A$ and Prohorov then $P(Y)$ is absolute $G_{\delta}$,

(2) if $P(Y)$ is absolute $G_{\delta \sigma}$ then $P(Y)$ is absolute $G_{\delta}$.

(3) if $P(Y)$ is absolute $F_{\sigma}$ then $P(Y)$ is absolute closed.

The necessary facts concerning absolute Borel class can be found in [2, p. 339].

II. Background. Two background theorems require explicit mention. The first is due to Prohorov and may be found in [4, p. 46, Theorem 6.5].

\footnotetext{
Received by the editors, November 20, 1981 and, in revised form. July 21, 1982. 1980 Mathematics Subject Classification. Primary 60B05, 60B10, 54F65, 54H05.

${ }^{1}$ The results contained in this paper were obtained by the author while he was a student of Jack B. Brown at Auburn University.
} 
Theorem A (Prohorov). If $X$ is separable and metric, then $P(X)$ is topologically complete iff $X$ is.

The second may be found in $[6$, p. 430].

THEOREM B. If $X$ is complete, then $F \subseteq X$ is topologically complete iff $F$ is a $G_{\delta}$ subset of $X$.

In [7] Varadarajan notes that a basis for the topology of weak convergence is formed by finite intersections of $\left\{m \in P(X): m\left(U^{\prime}\right)>m_{0}(U)-\varepsilon\right\}$ where $m_{0}$ is some fixed probability measure, $U$ is open in $X$ and $\varepsilon>0$. For our purposes we need to show that sets of a more restrictive form constitute a basis for $P(X)$.

Lemma 1. Sets of the form $N^{*}\left(m_{0} ; \varepsilon ; U_{1} \ldots, U_{n}\right)=\left\{m \in P(X): m\left(U_{i}\right)>m_{0}\left(U_{1}\right)\right.$ $-\varepsilon, i=1, n\}$ where $\varepsilon>0$ and $U_{1} \ldots, U_{n}$ are disjoint open subsets of $X$ such that $\sum_{1=1}^{n} m_{0}\left(U_{1}\right)=1$. form a basis for the weak topologi on $P(X)$.

Proof. The usual basis for the weak topology on $P(X)$ is defined by sets of the form $N\left(m_{0} ; \varepsilon ; f_{1} \ldots, f_{n}\right)=\left\{m \in P(X):\left|\int f_{1} d m-\int f_{1} d m_{0}\right|<\varepsilon, j=1, n\right\}$ where the functions $f$, are required to be bounded continuous functions from $X$ into the reals (see [4]). For convenience assume that the $f$, are into [0, 1].

As $N\left(m_{0} ; \varepsilon ; f_{1} \ldots f_{n}\right)=\bigcap_{1=1}^{n} N\left(m_{0} ; \varepsilon ; f_{1}\right)$, we need only consider $N\left(m_{0}: \varepsilon: f_{1}\right)$ and show that intersections may be accommodated.

Consider $N\left(m_{0} ; \varepsilon ; f\right)$ where $f$ is taken to be from $X$ into $[0,1]$. Pick an integer $n>2$ so that $(1 / n)<(\varepsilon / 8)$. Pick numbers $0<a_{0}<a_{1}<\cdots<a_{n+1}=1$ so that the mesh of the subdivision is less than $(1 / n)$ and $m_{0}\left(\left[f \in\left\{a_{0}, \ldots, a_{n}\right\}\right]\right)=0$. Note that the last property can be obtained since $n+1$ points are chosen and only countably many points are the image under $f$ of sets of positive $m_{0}$-measure.

Let $U_{0}=\left[f \in\left[0, a_{0}\right)\right] . \quad U_{1}=\left[f \in\left(a_{1}, 1, a_{1}\right)\right]$ for $1 \leqslant i \leqslant n$ and $U_{n+1}=[f \in$ $\left.\left(a_{n} .1\right]\right]$. Note $\sum_{1}^{n+1}{ }_{0} m_{0}\left(U_{1}\right)=1$. Pick $0<\delta<1$ so that $\delta(n+1)<(\varepsilon / 8)$.

Consider $m \in N^{*}\left(m_{0}:\left(\delta / 2^{n+1}\right) ; U_{0} \ldots . U_{n+1}\right)$. If $m\left(U_{n+1}\right) \geqslant m_{0}\left(U_{n+1}\right)+\delta$ then

$$
\begin{aligned}
\sum_{1}^{n+1} m\left(U_{1}\right) & \geqslant \sum_{1=0}^{n}=\left[m_{0}\left(U_{1}\right)-\delta / 2^{n+1}\right]+m_{0}\left(U_{n+1}\right)+\delta \\
& =1-\left[(n+1) / 2^{n+1}\right] \delta+\delta>1 .
\end{aligned}
$$

Clearly this holds for each $U_{1}$, so $\left|m\left(U_{1}\right)-m_{0}\left(U_{1}\right)\right|<\delta$ for $0 \leqslant i \leqslant n+1$. This gives the inequalities

$$
\sum_{1}^{n+1} m_{0}\left(U_{1}\right) a_{1} \leqslant \int f d m_{0} \leqslant \sum_{1}^{n+1} m_{0}\left(U_{1}\right) a_{1}
$$

and

$$
\sum_{1=1}^{n+1}\left[m_{0}\left(U_{1}\right)-\delta\right] a_{1}, \leqslant \int f d m \leqslant \sum_{1}^{n+1}\left[m_{0}\left(U_{1}\right)+\delta\right] a_{1} .
$$


Therefore

$$
\begin{aligned}
{\left[m_{0}\left(U_{0}\right)+\delta\right]\left(-a_{0}\right)-\sum_{i=1}^{n+1}\left[m_{0}\left(U_{i}\right)\left(a_{i}-a_{1-1}\right)+\delta a_{1}\right] \leqslant \int f d m_{0}-\int f d m } \\
\quad \leqslant m_{0}\left(U_{0}\right) a_{0}+\sum_{i=1}^{n+1}\left[m_{0}\left(U_{i}\right)\left(a_{1}-a_{i-1}\right)+\delta a_{1-1}\right] .
\end{aligned}
$$

Now $a_{0}<(\varepsilon / 8)$ implies $m\left(U_{0}\right) a_{0}<(\varepsilon / 8)$ and $\delta a_{0}<(\varepsilon / 8)$. Furthermore.

$$
\sum_{i=1}^{n+1} m_{0}\left(U_{i}\right)\left(a_{i}-a_{i-1}\right) \leqslant \frac{1}{n} \sum_{i=1}^{n+1} m\left(U_{i}\right) \leqslant \frac{1}{n}<\frac{\varepsilon}{8}
$$

and

$$
\sum_{i=1}^{n+1} \delta a_{i-1}<\sum_{i=1}^{n+1} \delta a_{i}=\delta \sum_{i=1}^{n+1} a_{i}<\delta(n+1)<\frac{\varepsilon}{8} .
$$

So $\left|\int f d m_{0}-\int f d m\right|<\varepsilon$.

In order to accommodate

$$
N\left(m_{0} ; \varepsilon ; f_{i}\right) \cap N\left(m_{0} ; \varepsilon ; f_{j}\right)
$$

consider $N^{*}\left(m_{0} ; \delta_{1} ; U_{1}, \ldots, U_{n}\right)$ and $N^{*}\left(m_{0} ; \delta_{2} ; O_{1}, \ldots, O_{k}\right)$. Let $U_{i j}=U_{1} \cap O$, and $\delta=\left(\min \left\{\delta_{1}, \delta_{2}\right\} / \max \{k, n\}\right)$. Note that for $m \in P(X), m\left(U_{1}\right) \geqslant \sum_{l=1}^{k} m\left(U_{1}\right)$ and $m\left(O_{j}\right) \geqslant \sum_{i=1}^{n} m\left(U_{i j}\right)$ and for $m_{0}$ these are equalities.

Suppose $m \in N^{*}\left(m_{0} ; \delta ; U_{11}, \ldots, U_{n k}\right)$. So $m\left(U_{i j}\right)>m_{0}\left(U_{i j}\right)-\delta$ implies

$$
\begin{aligned}
m\left(U_{i}\right) & \geqslant \sum_{j=1}^{k} m\left(U_{i j}\right)>\sum_{j=1}^{k}\left[m_{0}\left(U_{i j}\right)-\delta\right] \\
& =m_{0}\left(U_{i}\right)-k \delta \geqslant m_{0}\left(U_{i}\right)-\delta_{1} .
\end{aligned}
$$

Similarly $m\left(O_{j}\right)>m_{0}\left(O_{j}\right)-\delta_{2}$, so $m$ belongs to the intersection of $N^{*}\left(m_{0} ; \delta_{1} ; U_{1}, \ldots, U_{n}\right)$ and $N^{*}\left(M_{0} ; \delta_{2} ; O_{1}, \ldots, O_{k}\right)$.

$N^{*}\left(m_{0} ; \varepsilon ; U_{1}, \ldots, U_{n}\right)$ is open since it is equal to

$$
\bigcap_{i=1}^{n}\left\{m \in P(X): m\left(U_{i}\right)>m_{0}\left(U_{i}\right)-\varepsilon\right\}
$$

and these sets are open.

The following lemma follows directly from the Baire Category Theorem. It is stated as a lemma in the form that will be used in the example to follow.

Lemma 2. Suppose $X$ is topologically complete, $V$ is a closed subset of $X, O \subseteq V$ open relative to $V, N \subseteq V$ that is first category in $V$ and $\left\langle K_{i}\right\rangle$ is a sequence of closed sets in $X$ such that $O-N \subseteq \cup_{i=1}^{\infty} K_{i}$. There exists a set $U \subseteq O$ with the following properties:

(i) $U$ is open relative to $O$,

(ii) $\bar{U} \subseteq O(\bar{U}$ denoting the closure of $U$ in $X)$,

(iii) $\bar{U} \subseteq K_{j}$ for some $j$,

(iv) the diameter of $\bar{U}<\left(1 / 2^{i}\right)$ where $i$ is an arbitrary natural number. 
III. Main result. We now introduce the following notation. Let $X$ be a perfect, complete, separable metric space and $Y$ a countable dense subset of $X$. Let $Y=\left\{y_{1}, y_{2}, \ldots\right\}$ and $Y_{1}=\left\{y_{1}, y_{2}, \ldots, y_{1}\right\}$. Let $i_{1}, i_{2}, i_{3}, \ldots, i_{n}$ denote an increasing sequence of positive integers, $C_{1,}=\left\{m \in P(X) \mid m\left(Y_{1}\right) \geqslant 1-(1 / j)\right\}, \quad V_{n}=$ $\cap_{h}^{n},_{1} C_{1 k}$ and $C_{1}=\cup_{1=1}^{\infty} C_{1}$. Note that $C_{1}$ is closed in $P(X)$ and $\cap_{1,1}^{\infty} C_{1}=P(Y)$. Assume that $P(X)$ has been equipped with a complete metric.

LEMMA 3. $V_{n} \cap C_{i n+1}$ is nowhere dense in $V_{n}$.

Proof. $V_{n} \cap C_{1 n+1}$ is closed in $V_{n}$ so it remains to show that if $m_{0} \in V_{n} \cap C_{m+1}$ then any neighborhood of $m_{0}$ contains a point of $V_{n}-C_{1 n+1}$.

Consider $N^{*}\left(m_{0} ; \varepsilon ; U_{1}, \ldots, U_{k}\right)$. Since $X$ is perfect and complete and $Y$ is countable we may choose $a_{j}$ such that $a, \in U,-Y$ for $1 \leqslant j \leqslant k$.

Case 1. $m_{0}\left(Y_{t_{n}}\right)<1-1 /(n+1)$. Define $m \in P(X)$ by the following: $m\left(\left\{y_{1}\right\}\right)=$ $m_{0}(\{y\}$,$) for y, \in Y_{i_{n}}$ and $m\left(\left\{a_{j}\right\}\right)=m_{0}\left(U_{j}\right)-m\left(U, \cap Y_{i_{n}}\right)$ for $1 \leqslant j \leqslant k$. Clearly $m\left(U_{,}\right)=m_{0}\left(U_{,}\right)$and $m$ agrees with $m_{0}$ on $Y_{\iota_{n}}$ but $m(Y)<1-1 /(n+1)$. So $m \in N^{*}\left(m_{0} ; \varepsilon ; U_{1}, \ldots U_{k}\right) \cap\left[V_{n}-C_{1 n+1}\right]$.

Case 2. $m_{0}\left(Y_{t}\right) \geqslant 1-(1 / n+1)$. Let $t$ denote the first integer between 1 and $i_{n}$ inclusive with the property that $\Sigma_{1=1}^{\prime} m_{0}\left(\left\{y_{,}\right\}\right) \geqslant 1-1 /(n+1)$. Pick $0<r<1$ so that

$$
1-\frac{1}{n}<\sum_{j=1}^{t-1} m_{0}\left(\left\{y_{j}\right\}\right)+r m_{0}\left(\left\{y_{t}\right\}\right)<1-\frac{1}{n+1} .
$$

Define $m$ by the following: $m\left(\left\{y_{,}\right\}\right)=m_{0}\left(\left\{y_{1}\right\}\right)$ for $j<t, m\left(\left\{y_{t}\right\}\right)=r m_{0}\left(\left\{y_{t}\right\}\right)$ and $m(\{a\})=,m_{0}\left(U_{l}\right)-m\left(U_{,} \cap Y_{t}\right)$ for $1 \leqslant j \leqslant k$. Now, $m\left(U_{j}\right)=m_{0}\left(U_{,}\right)$and $m$ agrees with $m_{0}$ on $Y_{t-1}$. Since $m_{0} \in V_{n}, m\left(Y_{l,}\right) \geqslant 1-(1 / j)$ for $i,<t$. If $t \leqslant i, \leqslant i_{n}$ then $m\left(Y_{1}\right) \geqslant 1-(1 / n) \geqslant 1-(1 / j)$. Hence, $m \in V_{n}$. On the other hand, $m(Y)<1-$ $1 /(n+1)$ implies that $m \notin C_{i n+1}$. This completes the proof.

Lemma 4. $V_{n} \cap C_{n+1}$ is dense in $V_{n}$.

Proof. Let $m_{0} \in V_{n}$ and consider $N^{*}\left(M_{0} ; \varepsilon ; U_{1}, U_{2}, \ldots, U_{k}\right)$. Since $Y$ is dense and $X$ is perfect, $\left(Y-Y_{t_{n}}\right) \cap U_{J} \neq 0$. Pick $i>i_{n}$ with the property that $\left(Y_{t}-Y_{t_{n}}\right) \cap U_{\text {, }}$ $\neq 0,1 \leqslant j \leqslant k$. Pick $a_{j} \in\left(Y_{1}-Y_{i_{n}}\right) \cap U_{j}$. Define $m$ by the following: $m\left(\left\{y_{j}\right\}\right)=$ $m_{0}\left(\left\{y_{j}\right\}\right)$ for $1 \leqslant j \leqslant i_{n}$ and $m\left(\left\{a_{j}\right\}\right)=m_{0}\left(U_{j}\right)-m\left(Y_{i_{n}} \cap U_{j}\right)$. Clearly $m\left(Y_{\imath}\right)=1$, so $m \in C_{n+1}$. Furthermore $m$ agrees with $m_{0}$ on $Y_{i_{n}}$ implies $m \in V_{n}$ and $m\left(U_{j}\right)=m_{0}\left(U_{j}\right)$ implies $m \in N^{*}\left(m_{0} ; \varepsilon ; U_{1}, \ldots, U_{k}\right)$.

EXAMPLE 5. In this example the notation introduced before Lemma 3 will be used. It will be shown that $P(Y)$ is not a $G_{\delta \sigma}$ subset of $P(X)$. Assume that $P(X)$ has been equipped with complete metric.

Suppose $P(Y)$ is a $G_{\delta \sigma}$ subset of $P(X)$. Then $P(X)-P(Y)$ is an $F_{\sigma \delta}$ subset of $P(X)$ and there exist closed sets $\left\langle K_{i j}\right\rangle$ such that $P(Y)^{c}=\cap_{j=1}^{\infty} K_{j}$ where $K_{j}=$ $\cup_{i=1}^{\infty} K_{i j}$.

Using Lemmas 2, 3 and 4 a sequence of sets, $\left\langle\bar{O}_{n}\right\rangle$, will be constructed that have the property that their intersection belongs to both $P(Y)$ and $P(Y)^{c}$. Recall the notation introduced before Lemma 3. 
Let $i_{1}=1$. Then $V_{1}=C_{i_{1} 1}$ is a closed subset of $P(X)$. By Lemma 3 we have that $V_{1} \cap C_{2}$ is a first category $F_{\sigma}$ set with respect to $V_{1}$. Let $O_{1}=P(X)$. Now $O_{1}-\left(V_{1} \cap C_{2}\right) \subseteq C_{2}^{c} \subseteq P(Y)^{c} \subseteq K_{1}$ (note $O_{1} \subseteq V_{1}$ and in general $O_{n}$ will be a subset of $V_{n}$ ). By Lemma 2 there exists a set $U_{1} \subseteq O_{1}$ having properties (i)-(iv) as stated in the lemma. For property (iv) require that the diameter of $\bar{U}_{1}<\frac{1}{2}$. Note that $K_{1}$ corresponds to the union of the $K_{i}$ of Lemma 2.

Since $U_{1}$ is open in $O_{1}$ and, by Lemma $4, C_{2}$ is dense in $V_{1}$, we can pick $i_{2}>i_{1}$ so that $C_{i, 2}$ intersects $U_{1}$. Let $V_{2}=V_{1} \cap C_{i, 2}$ and $O_{2}=U_{1} \cap V_{2}$. Note that $O_{2}$ is open in $V_{2}$ and $V_{2}$ is a closed subset of $P(X)$ which is complete. Proceed to the $n+1$ step.

Assume that $i_{1}<\cdots<i_{n}$ have been defined, $V_{n}=\cap_{k=1}^{n} C_{1, k}, O_{1} \supseteq O_{2} \cdots O_{n}$ have been defined so that the diameter of $O_{k}<\left(1 / 2^{k-1}\right)$ and $O_{k} \subseteq V_{k}$ is open relative to $V_{k}$. By Lemmas 3 and $4, V_{n} \cap C_{n+1}$ is a first category $F_{\sigma}$ subset of $V_{n}$ and is dense in $V_{n}$. $O_{n}-\left(V_{n} \cap C_{n+1}\right) \subseteq C_{n+1}^{c} \subseteq P(Y)^{c} \subseteq K_{n}$, so by Lemma 2 there exists $U_{n} \subseteq O_{n}$ having properties (i)-(iv) (where the diameter of $\bar{U}_{n}<\left(1 / 2^{n}\right)$ ). Pick $i_{n+1}>i_{n}$ so that $C_{i_{n, 1} n+1}$ intersects $U_{n}$. Let $V_{n+1}=V_{n} \cap C_{i_{n, 1} n+1}$ and $O_{n+1}=U_{n} \cap$ $V_{n+1}$.

The sets, $\bar{O}_{1} \supseteq \bar{O}_{2} \supseteq \cdots$, are nonempty and the diameter of $\bar{O}_{n}<\left(1 / 2^{n-1}\right)$ for $n>1$. Since $P(X)$ is complete there exists $m \in \cap_{n=1}^{x} \bar{O}_{n}$. For each $n, O_{n} \subseteq V_{n}$ and $V_{n}$ is closed so $\bar{O}_{n} \subseteq V_{n}$ and $m \in V_{n}$. Therefore, for each $n, m \in C_{i_{n} n}$. so $m \in C_{n}$ and $m \in \cap_{n=1}^{x} C_{n}=P(Y)$.

On the other hand $\bar{O}_{n} \subseteq \bar{U}_{n-1}$ for $n \geqslant 2$. By property (iii) of Lemma 2. $\bar{U}_{n-1} \subseteq$ $K_{n-1}$. This gives that $m \in K_{n}$ for each $n$ and $m \in \cap_{n=1}^{x} K_{n}=P(Y)^{c}$.

THEOREM 6. If $X$ is separable metric and coanalytic but not topologically complete. then $X$ contains a countable, dense in itself, $G_{\delta}$ subspace.

Proof. See [1 or 5].

THEOREM 7. If $X$ is a complete separable metric space and $Y \subseteq X$ and $P(Y)$ is a $G_{\delta \text { o }}$ subset of $P(X)$ then $P(Y)$ is in fact a $G_{\delta}$ subset of $P(X)$.

Proof. Suppose $Y$ fails to be a $G_{\delta}$ subset of $X$ (and hence, by Theorem B, $Y$ is not topologically complete). $Y$ and $X$ are homeomorphic to the degenerate measures (point masses) in $P(Y)$ and $P(X)$, see [4], so $Y$ is a $G_{\delta \sigma}$ subset of $X$. Since $X$ is complete, $Y$ is coanalytic. By Theorem 6 there exists a $G_{\delta}$ subset of $X$. call it $G$, such that $G \cap Y$ is countable and dense in itself. Let $Y_{1}=G \cap Y$ and $X_{1}=\bar{Y}_{1} \cap G$, i.e. $X_{1}$ is the closure of $Y_{1}$ in $G . X_{1}$ is dense in itself and topologically complete. $Y_{1}$ is a countable dense subset of $X_{1}$.

Since $Y_{1}=Y \cap X_{1}$ we have that $P\left(Y_{1}\right)=P(Y) \cap P\left(X_{1}\right)$. By hypothesis $P(Y)$ is a $G_{\delta \sigma}$ in $P(X)$, so $P\left(Y_{1}\right)$ is a $G_{\delta \sigma}$ in $P\left(X_{1}\right)$. On the other hand, $X_{1}$ and $Y_{1}$ have the properties specified in Example 5 in which it was shown that $P\left(Y_{1}\right)$ is not a $G_{\delta \sigma}$ in $P\left(X_{1}\right)$; hence, $Y$ must be a $G_{\delta}$ subset of $X$ and therefore, by Theorems A and B. $P(Y)$ is a $G_{\delta}$ subset of $P(X)$.

RemarK. It is not difficult to show that if $Y$ belongs to the $\alpha$ th multiplicative Borel class with respect to $X$, then $P(Y)$ belongs to the $\alpha$ th multiplicative Borel class with respect to $P(X)$. In light of statements (2) and (3) of $\S I$, is the following true? 
If $P(Y)$ belongs to the $\beta$ th, $\beta>0$, additive Borel class with respect to $P(X)$, then there exists $\alpha<\beta$ such that $P(Y)$ belongs to the $\alpha$ th multiplicative Borel class with respect to $P(Y)$.

\section{REFERENCES}

1. W. Hurewicz. Relativ perfekte Teile von punktenmengen und mengen. Fund. Math. 12 (1928), $78-109$

2. C. Kuratowski. Topologie. I. 4th ed., Monografie Mat.. Warsaw. 1958.

3. N. Y. Luther. Locally compact spaces of measures. Proc. Amer. Math. Soc. 25 (1970). $541-547$.

4. K. R. Pathasarthy. Prohability measures on metric spaces. Academic Press. New York and London. 1967.

5. D. Preiss. Metric spaces in which Prohorov's theorem is not ealid. Z. Wahrsch. Veru. (icbicte 27 (1973). 109-116.

6. K. Kuratowski. Topologi. I. Academic Press. New York and London. 1966.

7. V. S. Varadarajan. Measures on topological spaces. Amer. Math. Soc. Transl. (2) 48 (1965). 161-228.

TRW Systems 526/610. P. O. Box 1310. San Bernardino. California 92402 